\title{
A NOTE ON INTERSECTIONS
}

\author{
E. MICHAEL ${ }^{1}$
}

1. Introduction. In this paper, we partialiy extend to higher cardinals a set-theoretic result of N. A. Sanin $[3 ; 5]$, and use it to generalize a result of $\mathrm{R}$. S. Pierce [2] on intersections of cubes in product spaces. $^{2}$

Call a collection $S$ of sets quasi-disjoint if each $x \notin \cap S$ is in at most one $S \in \delta$. Denote the cardinality of a set $A$ by $|A|$.

THEOREM 1.1. If $S$ is a collection of sets all having cardinality $\leqq s$ $(s>0)$, and if each quasi-disjoint subcollection of $\mathcal{S}$ has cardinality $\leqq r$ (infinite), then $|s| \leqq r^{s}$.

For $s$ finite, this result was obtained by Sanin $[3 ; 5] .^{3}$

By a cube in a product space $X=\prod_{\alpha \in \mathrm{A}} X_{\alpha}$, we mean a set $V=\prod_{\alpha \in \mathbf{A}} V_{\alpha}$ with $V_{\alpha} \subset X_{\alpha}$. If $V$ is a cube, $A_{V}$ will denote $\left\{\alpha \in \mathbf{A} \mid V_{\alpha} \neq X_{\alpha}\right\}$. The letters $u, v$, and $w$ will denote cardinals, not all finite.

TheOREM 1.2. Let $X=\prod_{\alpha \in \mathrm{A}} X_{\alpha}$, with $\left|X_{\alpha}\right| \leqq u$ for all $\alpha$, and let $v$ be a collection of cubes in $X$ with $\left|\mathrm{A}_{V}\right| \leqq v$ for all $V \in V$. If every point of $X$ is in $\leqq w$ elements of $v$, then $|v| \leqq(u w)^{v}$.

In the special case where $w=1$ (which makes $v$ disjoint), Theorem 1.2 was essentially obtained by Pierce [2], whose proof (based on an idea of H. H. Corson) influenced the proof of our Theorem 1.1.

2. Proof of Theorem 1.1. Let $\tau$ be the smallest ordinal of cardinal-

Received by the editors August 8, 1960 and, in revised form, February 8, 1961. tion.

1 Supported by grants from the O.N.R., the N.S.F., and the Guggenheim Founda-

2 Added in Proof. Paul Erdös has kindly pointed out that a theorem equivalent to Theorem 1.1 was obtained by him and R. Rado in [Intersection theorems for systems of sets, J. London Math. Soc. 35 (1960), 85-90, Theorem I (ii)]. Their proof, while actually yielding some information even for finite cardinals $r$, is much longer than the one given here, which provided a reason for going ahead with the publication of this paper.

3 Sanin's principal application of his result was to show $[4 ; 5]$ that, for infinite cardinals $r$, the following topological property $\breve{S}(r)$ is preserved under arbitrary cartesian products: Every collection of open sets of cardinality $>r$ has a subcollection of cardinality $>r$ with nonempty intersection. (This need only be shown for open cubes, where it is first proved for finite products, after which the general case is proved like our Theorem 1.2.) For $\zeta\left(k_{0}\right)$ (which is weaker than separability, although equivalent to it in metric spaces), Sanin's theorem should be compared with the results in [1]. 
ity $>s$. For each $\nu<\tau$, we will construct a subcollection $S$, of $S$ such that

$$
\left|S_{\nu}\right| \leqq r^{2}
$$$$
\text { for all } \nu<\tau \text {. }
$$

$$
S=U_{\nu<r} S_{\nu} \text {. }
$$

This will suffice, since then $|\delta| \leqq 2^{8} r^{s}=r^{s}$.

For convenience, we begin our inductive definition of $S_{\nu}$ by letting $\delta_{-1}=\phi$. Suppose now that $\delta_{\mu}$ has been defined for $\mu<\nu$, and let us define $\delta_{v}$. Let

$$
E_{\nu}=U_{\mu<\nu}\left(\cap S_{\mu}\right)
$$

For each $K \subset E_{p}$, let

$$
\mathfrak{K}=\left\{S \in \mathcal{s} \mid S \cap E_{\nu}=K\right\},
$$

and let $\mathcal{K}^{*}$ be a maximal, quasi-disjoint subcollection of $\mathscr{K}$; if there exist $S, T \in \Re$ with $S \cap T=K$, then we pick $\mathscr{K}^{*}$ to contain such an $S$ and $T$, so that

$$
\cap \mathcal{K}^{*}=K
$$

Finally, let

$$
\mathrm{S}_{\nu}=\cap\left\{\mathfrak{K}^{*} \mid K \subset E_{\nu}\right\} .
$$

Let us verify (a) and (b).

To verify (a), note first that $\left|E_{\nu}\right| \leqq s s r^{s}=r^{*}$ by (a) for cardinals $<\nu$. Hence $E$, has at most $\left(r^{s}\right)^{s}=r^{s}$ subsets $K$ of cardinality $\leqq s$, and since each $\mathcal{K}^{*}$ has cardinality $\leqq r^{8}$, we have $\left|S_{\nu}\right| \leqq r^{8} r^{8}=r^{8}$.

To verify (b), suppose that, on the contrary, there exists an $S \in \mathcal{S}$ which is not in any $\mathcal{S}_{\boldsymbol{v}}$. We will get a contradiction by finding distinct points $x_{\nu}$ in $S$ for all $\nu<\tau$; this is impossible, since $|S| \leqq s$, while $|\tau|>s$. To define $x_{\nu}$, let $K_{\nu}=S \cap E_{\nu}$, pick any $T_{\nu} \in \mathcal{K}_{\nu}^{*}$, and let $x_{\nu}$ be any element of $S \cap\left(T_{\nu}-E_{v}\right)$ : this set cannot be empty, for if it were, we would have $S \cap T_{\nu}=K_{v}$, whence $\cap \mathcal{K}_{\nu}^{*}=K_{\nu}$ by (3), and thus $\varkappa_{*}^{*} \cap\{S\}$ would still be quasi-disjoint, contradicting the maximality of $\mathfrak{K}_{\nu}^{*}$. Now if $\mu<\nu<\tau$, then $x_{\mu} \in T_{\mu} \subset \mathrm{US}_{\mu} \subset E_{\nu}$, while $x_{\nu} \notin E_{\nu}$. Hence $x_{\mu} \neq x_{\nu}$, and the proof is complete.

3. An indexed Theorem 1.1. The following indexed version of Theorem 1.1 is useful for applications.

If $\left\{S_{\lambda} \mid \lambda \in \Lambda\right\}$ is an indexed collection of subsets of a set $E$, call $\Lambda^{\prime} \subset \Lambda$ regular if each $x \in E$ is in $S_{\lambda}$ either for all $\lambda \in \Lambda^{\prime}$ or for at most one $\lambda \in \Lambda^{\prime}$.

THEOREM 3.1. If $\left\{S_{\lambda} \mid \lambda \in \Lambda\right\}$ is an indexed collection of sets all having 
cardinality $\leqq s$, and if each regular $\Lambda^{\prime} \subset \Lambda$ has cardinality $\leqq r$ (infinite), then $\Lambda$ has cardinality $\leqq r^{8}$.

Proof. If $S=\left\{S_{\lambda} \mid \lambda \in \Lambda\right\}$, then all the assumptions of Theorem 1.1 are satisfied, so $|\delta| \leqq r^{s}$. For each $S \in \mathcal{S}$, let $\Lambda_{S}=\left\{\lambda \in \Lambda \mid S_{\lambda}=S\right\}$; then $\Lambda_{S}$ is regular, so has cardinality $\leqq r$. But $\Lambda=\bigcup\left\{\Lambda_{S} \mid S \in \mathcal{S}\right\}$, so $|\Lambda|$ $\leqq r r^{8}=r^{8}$.

4. Proof of Theorem 1.2. We apply Theorem 3.1 to the indexed collection $\left\{A_{V} \mid V \in V\right\}$. By Theorem 3.1, it suffices to show that each regular $u \subset \mathcal{V}$ has cardinality $\leqq w u^{*}$. So let $B=\cap\left\{A_{V} \mid V \in \mathcal{U}\right\}$. For each $V \in \mathcal{u}$, let $\pi(V)$ denote the projection of $V$ onto $\prod_{\alpha \in B} X_{\alpha}$. Now $|\mathrm{B}| \leqq v$, so $\left|\prod_{\alpha \in \mathrm{B}} X_{\alpha}\right| \leqq u^{v}$. Hence if $u$ had cardinality $>w u^{v}$, there would exist a $\mathscr{\complement} \subset \mathcal{U}$, with $|w|>w$, such that $\cap\{\pi(V) \mid V \in w\} \neq \varnothing$. But, keeping in mind that $u$ is regular, this would imply that $\cap w$ $\neq \varnothing$, which is impossible.

\section{REFERENCES}

1. E. Marczewski, Separabilité et multiplication cartesienne des espaces topologiques, Fund. Math. 34 (1947), 127-143.

2. R. S. Pierce, Some questions about complete Boolean algebras, Proceedings of Symposia in Pure Mathematics, Vol. II, Lattice Theory, Amer. Math. Soc., Providence, R. I., 1961.

3. N. A. S̉anin, $A$ theorem from the general theory of sets, C. R. (Doklady) Acad. Sci. URSS 53 (1946), 399-400.

4. - On intersection of open subsets in the product of topological spaces, C. R. (Doklady) Acad. Sci. URSS 53 (1946), 499-501. (Rnssian)

5. - On the product of topological spaces, Trudy Mat. Inst. Steklov 24 (1948), 112 pp. (Russian)

UNIVERSITY OF WASHINGTON AND The Institute for Advanced Study 